\section{Quasiperiodic order in dissipative systems}

SIR-The concept of quasicrystalline order $^{1}$ in relation to experimental observations $^{2-}$ in $\mathrm{Al}_{6} \mathrm{Mn}$ suggests that quasiperiodic structures are also likely to appear in driven systems undergoing patterning instabilities. (Classical examples are given by convective instabilities in fluids or Turing instabilities in chemical systems, for example, ref. 5). Despite the complexity of the dynamics, such symmetry-breaking instabilities may be described near threshold by a kinetic equation for the unstable or critical modes which play the role of an order parameter. They are related for example to the velocity field in hydrodynamical systems or to the concentration of active species in chemical systems ${ }^{h .7}$. The stabilities of the patterns may be ordered in decreasing values of the associated Lyapunov functional which plays the role of the GinzburgLandau-Brazovskii potential in equilibrium situations.

Near threshold, structures built on wavevectors of critical wavelength may be stable and when the Lyapunov functional is limited to its fourth order invariant a situation very similar to the Landau treatment of crystallization is recovered ${ }^{*}$. Among the possible patterns one finds, for example, layered structures corresponding to modulations in one direction only, bimodal patterns and structures with triangular or cubic symmetry corresponding to combinations of critical modes with wavevectors forming equilateral triangles?

However, we have to keep in mind that the kinetic equation for the order parameter-like variables is obtained via the adiabatic elimination of stable noncritical modes which are driven by the critical ones. Hence their contribution may become important for increasing values of the bifurcation parameter. As a result, the intensity of the coupling between three modes is renormalized and, furthermore, higher order nonlinearities are generated by the driven modes as in the multicomponent Landau theory ${ }^{4}$. The first nonlinear coupling induced by this effect in the kinetic equation involves four critical modes corresponding to a fifth order invariant in the Lyapunov functional ${ }^{3}$. This term favours the formation of structures built on wavevectors forming an equilateral pentagon which in two-dimensional systems has the symmetry of a Penrose lattice.

The emergence of high order nonlinearities in the dynamics of the critical modes favouring quasiperiodic patterns is a general feature of this description of systems undergoing symmetry-breaking instabilities ${ }^{6.7}$. Hence, according ot the intensities of the nonlinear couplings which are system dependent, quasiperiodic structures could become the stablest ones in some range of the parameters. Having a dominant growth rate versus layered structures they should also appear after sudden increases of the control parameter.

In various convective instabilities, the increase of the constraint beyond threshold leads to the nucleation of defects and ultimately to the destruction of the translational order". It was suggested that this effect could be related to a dislocation induced melting similar to what happens in two-dimensional solids ${ }^{4,10}$. From the present analysis, however, one may also expect the appearance of quasiperiodic structures well beyond threshold. It would thus be interesting to perform diffraction experiments for such systems after slow and sudden increases of the bifurcation parameter in the region where translational order is lacking to test whether the disorganization is related to a dislocation-induced melting or to the transition from periodic to quasiperiodic structures.

The authors are research associates of the Belgian National Fund for Scientific Research.

Service de Chimie-Physique II.

Université Libre de Bruxelles, CP 231,

$B$-I050 Brussels, Belgium

1. Maddox, J. Nalure 314, 575, (1985).

2. Shechtman, D.. Bleck, L., Gratias, D., Chan, J.W. Phys. Rev. Lett. 53 1951, (1984).

Bak, P. Phys. Rev. Lett. 54, 1517, (1985).

4. Mermin, N.D. \& Troian, S.M. Phys. Rev. Lett. 54, 1524. (1985)

Wesfreid, J.E. \& S. Zaleski, Cellular Structures in Instabilities, Lecture Notes in Physics 210 eds (Springer, Berlin). ties, Lecture Notes in Physics 210 eds (Springer, Berlin).

6. Swift, J, \& Hohenberg, P.C. Phys. Rev. A15, 319, (1977).
7. Walgraef, D., Dewel, G. \& Borckmans, P. Adv. Chem. Walgraef, D., Dewel,
Phys. 49, 311, (1981).

8. Alexander, S. \& McTague, J.P. Phys. Rev. Lett. 41, 102. (1978).

Ocelli, R., Guazzelli, E. \& Pantaloni, J. J. de Physique Lett. 44, 1567, (1983).

10. Toner, J. \& Nelson, D. Phys. Rev, B23, 316, (1981).

\section{The cosmic connection of catastrophism?}

SIR-The remarks by Weissman' on the review by Maddox $^{2}$ require some additional comment. This relates to a possible extraterrestrial cause of terrestrial catastrophism and, in particular, to the origin of the suggestion that this might be associated with the Sun's motion in the Galaxy.

As previously pointed out in these pages ${ }^{3}$ pulsations are not a new idea and were suggested more than half-a-century ago $^{4}$. Later, in 1947, Umbgrove ${ }^{5}$ reasoned that the Earth had a periodicity of about $250 \mathrm{Myr}$ and drew attention to the similarity between this and the galactic day. Although Umbgrove was probably the first to make such a suggestion, the validity of much of his evidence can certainly now be questioned ${ }^{\text {. }}$.

Probably the first experimental measurements relevant to this topic were carried out in the early $1960 \mathrm{~s}^{7}$ and the re- sults were published in this journal in $1971^{\circ}$. These measurements indicated that the times of emplacement (in Myr) of mantle-derived carbonatites (for at least the last $1.7 \mathrm{Gyr}$ ) could be approximated very closely by the relationship $T_{n}=233 n$ -102 , with $n$ integral. The similarity of this period with that for galactic rotation was then noted. The significance of the data set used in deriving this conclusion has since been tested". From other evidence Innanen et al." have subsequently proposed a galactic model from which they derive an identical numerical value of 233 Myr for the length of the galactic year.

In 1971, plate tectonics was in its infancy and episodicity and global synchroneity were rather unfashionable concepts in geology. Over the years, this data set has therefore been continually expanded and improved" and incorporated into a hypothesis of Earth behaviour' ${ }^{2}$, compatible with plate tectonics, which nonetheless preserves this fundamental periodicity. This hypothesis is testable by carrying out more accurate age measurements on carbonatites.

One striking feature of the time distrivution is the near coincidence of times $T_{1}, T_{2}$ and $T_{3}$ with the boundaries of geological periods in Phanerozoic time ${ }^{13}$. It is therefore tempting to extrapolate backwards $(n \geqslant 4)$ for a Precambrian timescale. Another corollary of this hypothesis is that, as a large number of carbonatites exist with ages around $65 \mathrm{Myr}$ (see, for example, refs 12,14 ), an atmospheric spike of carbonatite-derived $\mathrm{CO}_{2}$ may have influenced events near the Cretaceous-Tertiary ${ }^{15}$ boundary. This temporal coincidence can again be tested by accurate geochronological measurements. Whether, in addition, widespread carbonatite emplacement can explain the well-documented geochemical anomalies associated with this boundary is currently being examined by analysing rocks from (dated) carbonatite complexes which were emplaced across the boundary.

\section{Scottish Universities Research}

\section{R. M. MACINTYRE} and Reactor Centre,

\section{East Kilbride,}

Glasgow G75 0QU, UK

I. Weissman, P.R. Nature 316, 572 (1985)

Maddox. J Nature 315.627 (1985).

Machado. F. Nature 214. 1317.1318 (1967)

4. Bucher, W.H. The Deformation of the Earth's Crust (University Press. Princeton. 1933).

Umbgrove. J.H.F. The Pulse of the Earth (Martinus Nijhoff. The Hague, 1947)

6. Harland, W.B. J. geol. Soc. Lond. 138. 197-203 (1981).

7. Macintyre. R.M. thesis. Univ. Toronto (1966).

8. Macintyre, R.M. Nature 230, 79-81 (1971).

. Rampino, M.R. Stothers. R.B. Science 226. 1427-1431 (1984).

10. Innanen K.A. Patrick A T \& Duley. W W Astrophys. Space Sci. 57.511-515(1978)

11. Macintyre. R.M. EOS 54. 239 (1973): 57. 675 (1976): in Proc. 2nd Int. Conf. Basement Teclonics (ed Podwysocki. M.H. \& Earle, J.L.) 386 (Newark. Delaware. 1976).

12. Macintyre. R.M. J. geol. Soc. Lond. 133. 375-384 (1977).

13. Macintyre, R.M. U.S.G.S. Open File Rpt. 78-701, 272-274 (1978).

14. Ulbrick, H.H.G.J. \& Gomes, C.B. Earth Sci Rev. 17 135-154 (1981)

15. Macintyre. R.M. ISCDS Newsletter 4, 2. 5-6(1981): Terra Cognita 2, 215 (1982) 